\title{
Adipocyte and lipid metabolism in cancer drug resistance
}

\author{
Yihai Cao \\ Department of Microbiology, Tumor and Cell Biology, Karolinska Institutet, Stockholm, Sweden.
}

\begin{abstract}
Development of novel and effective therapeutics for treating various cancers is probably the most congested and challenging enterprise of pharmaceutical companies. Diverse drugs targeting malignant and nonmalignant cells receive clinical approval each year from the FDA. Targeting cancer cells and nonmalignant cells unavoidably changes the tumor microenvironment, and cellular and molecular components relentlessly alter in response to drugs. Cancer cells often reprogram their metabolic pathways to adapt to environmental challenges and facilitate survival, proliferation, and metastasis. While cancer cells' dependence on glycolysis for energy production is well studied, the roles of adipocytes and lipid metabolic reprogramming in supporting cancer growth, metastasis, and drug responses are less understood. This Review focuses on emerging mechanisms involving adipocytes and lipid metabolism in altering the response to cancer treatment. In particular, we discuss mechanisms underlying cancer-associated adipocytes and lipid metabolic reprogramming in cancer drug resistance.
\end{abstract}

\section{Introduction}

Most types of cancer exploit lipid and cholesterol to meet their unlimited energy demands (1). Lipid catabolism is achieved through the fatty acid $\beta$-oxidation (FAO) pathway, using both exogenous and endogenous FAs $(2,3)$. In some nonglycolytic cancers, such as prostate cancer and lymphoma, lipid-dependent metabolism that supports cancer cell growth and metastasis becomes a prominent pathway for energy production (4-6). Cancer cells obtain lipids and lipoproteins through two mechanisms: uptake of exogenous lipids from their local microenvironment and de novo synthesis of endogenous lipid molecules (2). Cancer cells even possess some adipocyte characteristics, storing excess lipids in the form of lipid droplets, which supply energy to power their expansion and metastasis $(7,8)$.

Obesity, a global pandemic, is associated with an increased incidence of certain types of cancer, including breast cancer (BC), colorectal cancer (CRC), and pancreatic ductal cancer (PDAC) (9-11). Notably, obese adipocytes supply more fatty acids (FAs) to cancer cells than nonobese adipocytes, which increases the FAO-generated energy available for tumor growth and metastasis (12). Cancer-associated adipocytes (CAAs) in the tumor microenvironment (TME) play dynamic and sophisticated roles in facilitating tumor growth and drug responses $(13,14)$. CAAs provide fuel, growth factors, and cytokines and transdifferentiate into other stromal cells to alter tumor growth, metastasis, and drug responses. This Review highlights the impact of adipocytes and cancer lipid metabolism on cancer drug responses and discusses the emerging understanding of mechanisms underlying metabolic reprogramming in tumor responses to anticancer drugs.

Conflict of interest: YC is the founder and a stockholder of Clanotech, which develops therapeutics for ophthalmological disorders and fibrotic disease.

Copyright: (5) 2019, American Society for Clinical Investigation.

Reference information: J Clin Invest. 2019;129(8):3006-3017.

https://doi.org/10.1172/JCI127201.

\section{Cancer-associated adipocytes}

Since most cancer types arise in tissues and organs in association with white adipose tissue (WAT), this Review will hereafter use the term "adipocytes" in its discussion of white adipocytes. The broad definition of CAAs may include several types of adipocytes. Intratumoral adipocytes infiltrate into or are recruited to the tumor tissue. Together with other cells such as stromal fibroblasts, vascular cells, inflammatory cells, and immune cells, they constitute a portion of tumor mass (14). De novo differentiated adipocytes are preadipocytes and mature adipocytes that differentiate from mesenchymal stem cells within the tumor tissue. These cells may not exhibit discernible adipocyte features at their origin. However, they are influenced by factors in the TME to become adipocytes or adipocyte-like cells that store excessive lipid energy. After prolonged incubation with BC cells, mature adipocytes lose their lipid content and show fibroblast-like morphology, suggesting their contribution to cancer-associated fibroblast (CAF) expansion (15). Peritumoral adipocytes are a population of adipocytes that do not penetrate into the tumor tissue, but remain in close association with tumors in the surrounding area $(14,16)$. Remodeled adipocytes are created in response to cancer invasion, which remodels the extracellular matrix, separating adipose tissue from surroundings or engulfing a cluster of adipocytes to become part of the tumor tissue. This adipocyte-engulfing mechanism may exist in many cancer types, including BC, melanoma, ovarian cancer (OC), prostate cancer (PC), and CRC. Tumor-educated adipocytes are not in direct physical contact with cancer cells and may even reside in distal tissues and organs, yet tumor-produced growth factors and cytokines might influence their biological functions to facilitate tumor growth and spreading (17). Metastasis-associated adipocytes are created when metastatic cancer cells travel to distal organs that may transiently or enduringly contact adipocytes in remote tissues. For example, cancers such as BC and PC often metastasize to bone marrow (BM), where adipocytes are one of the most abundant cell types. BM adipocytes substantially 
Table 1. Examples of dipocyte-derived tumor-promoting endocrine hormones, growth factors, adipokines, and adipocytokines

\begin{tabular}{|c|c|c|c|}
\hline Factor & Function & Cancer and metastasis & Reference \\
\hline \multicolumn{4}{|l|}{ Hormone } \\
\hline Leptin & Angiogenesis, cancer cell, inflammation & Promotes tumor growth, metastasis & 30 \\
\hline Resistin & Angiogenesis, cancer cell, inflammation & Promotes tumor growth, metastasis & 31 \\
\hline \multicolumn{4}{|l|}{ Adipokine } \\
\hline Adipsin & Tumorigenesis & Promotes tumor growth, metastasis & 32 \\
\hline Visfatin & Angiogenesis, cancer cell & Promotes tumor growth, metastasis & 33 \\
\hline Chemerin & Tumorigenesis & Promotes tumor growth, metastasis & 34 \\
\hline \multicolumn{4}{|l|}{ Adipocytokine } \\
\hline IL-6 & Inflammation, angiogenesis & Promotes tumor growth, metastasis & 35 \\
\hline IL-1 $\beta$ & Inflammation, angiogenesis & Promotes tumor growth, metastasis & 36 \\
\hline TNF- $\alpha$ & Inflammation, lymph- and angiogenesis & Promotes tumor growth, metastasis & 37 \\
\hline CCL2 (MCP-1) & Inflammation, tumorigenesis & Promotes tumor growth, metastasis & 38 \\
\hline \multicolumn{4}{|l|}{ Growth factor } \\
\hline VEGF & Angiogenesis & Promotes tumor growth, metastasis & 39 \\
\hline BMP4 & Cancer cell proliferation and migration & Promotes tumor growth, metastasis & 40 \\
\hline HGF & Tumorigenesis, fibrosis & Promotes tumor growth, metastasis & 41 \\
\hline IGF-1 & Tumorigenesis, cancer cell survival & Promotes tumor growth, metastasis & 42 \\
\hline TGF- $\beta$ & Tumorigenesis, fibrosis, angiogenesis & Promotes tumor growth, metastasis & 43 \\
\hline \multicolumn{4}{|l|}{ Protease } \\
\hline MMP1 & Extracellular matrix degradation & Promotes invasion, metastasis & 44 \\
\hline MMP2 & Extracellular matrix degradation & Promotes invasion, metastasis & 44 \\
\hline MMP11 & Extracellular matrix degradation & Promotes invasion, metastasis & 44 \\
\hline
\end{tabular}

modulate metastatic tumor growth (18). A narrower definition of CAAs may only include intratumoral and peritumoral adipocytes.

Intratumoral and peritumoral adipocytes make direct contact with malignant and nonmalignant cells in the TME. Reciprocal interactions between adipocytes, cancer cells, and other stromal cells are mediated by paracrine factors and cell surface molecules. CAAs produce a range of growth factors, adipokines, and adipocytokines to affect cancer cell growth, survival, and migration. For example, CAAs frequently release fibroblast growth factors (FGFs), leptin, adiponectin, IL-1 $\beta$, IL-6, TNF- $\alpha$, CCL2, and CCL5 (19-22). Conversely, cancer cells produce signaling molecules that could trigger lipolysis in intratumoral adipocytes and peritumoral adipocytes, and even induce a global lipolysis program that affects all adipose depots in the body. For example, tumor-derived TNF- $\alpha$ and IL-6 induce adipose atrophy, which manifests as cancer cachexia, a common systemic disease in human cancer patients (23). Adipose and muscular atrophy in cachexia represents imbalanced energy metabolism, also called wasting syndrome. Recent findings show that WAT browning dissipates energy, substantially contributing to cachexia development (24). In cancer patients, cachexia jeopardizes quality of life and tolerance to cancer drugs. In fact, a large number of cachexia patients may die of treatment rather cancer disease.

\section{CAAs in tumor growth and metastasis}

Accumulating experimental evidence shows that adipocytes considerably influence tumor cell behavior by stimulating proliferation, migration, and survival (25-27). Several possible mechanisms underlying adipocyte-facilitated tumor development and progression have emerged, including (a) supply of lipids for cancer cell membrane and cellular organelle constitution $(28,29)$; (b) release of endocrine hormones, growth factors, adipokines, and adipocytokines (refs. 30-44 and Table 1); (c) metabolic reprogramming in cancer cells $(2,3)$; (d) protease production necessary for cancer invasion $(45,46)$; and (e) recruitment of nonadipocyte stromal cells such as inflammatory cells and vascular cells $(47,48)$. The complex and dynamic functions of CAAs in modulating tumor growth and metastasis are summarized in Figure 1.

In the presence of CAAs, cancer cells may switch their metabolic program from glycolysis to lipiddependent energy production, which is required for tumor growth, invasion, and metastasis (2). CAAs also produce various proteases to facilitate cancer invasion and metastasis. For example, CAAs in $\mathrm{BC}$ express high levels of metalloproteinase 11 (MMP11, also known as stromelysin-3) to facilitate cancer cell invasion in surrounding tissues $(49,50)$. As discussed above, adipocytes produce a range of adipocytokines and adipokines that recruit and activate inflammatory cells in the TME. Tumor-associated macrophages (TAMs), especially inflammatory M2 macrophages, promote cancer invasion and metastasis (51, 52). Recent findings show that adipocytes contribute substantially to tumor angiogenesis $(3,19,47)$, a crucial process for tumor growth and metastasis. Thus, CAAs play complex, dynamic, and miscellaneous roles in modulating tumor growth and metastasis (Figure 1). The healthy adipocytes located remotely from the tumor tissue could also heavily contribute to tumor growth by supplying lipid molecules, metabolites, and growth signals. Cancer cachexia-related adipose atrophy may represent the consequence of unceasing demand of lipid supply for tumor expansion.

\section{Cancer lipid metabolism}

Nearly all healthy cells produce energy through mitochondrial oxidative phosphorylation, i.e., transferring electrons from NADH or $\mathrm{FADH}_{2}$ to $\mathrm{O}_{2}$ via a series of electron carriers within mitochondria (53). In contrast to healthy cells, most cancer cells synthesize energy molecules through a high rate of glycolysis and the lactic acid fermentation pathway, i.e., the Warburg effect $(54,55)$. Although aerobic glycolysis produces ATP less efficiently than oxidative phosphorylation, this metabolic pathway generates additional metabolites that support tumor growth (56). Lipid metabolic reprogramming in cancer cells occurs using both de novo lipogenic synthesis (production of triglycerides/cholesterol) and the catabolic pathway. Similarly to adipocytes, some cancer cells store excessive energy as lipid droplets that can be further broken down into free fatty acids (FFAs) $(25,57)$. Glycolysis- and glutaminolysis- 


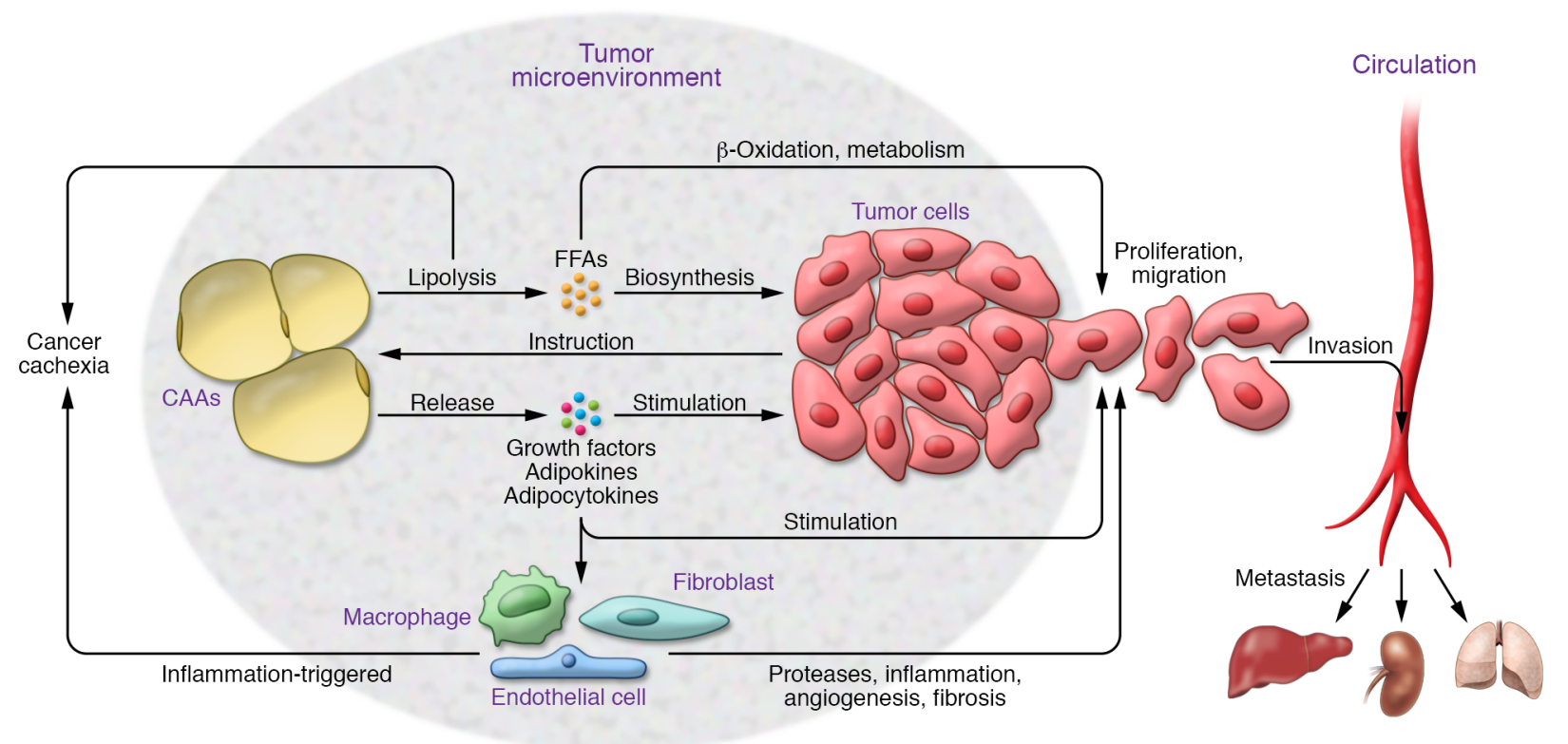

Figure 1. Mechanisms of cancer-associated adipocytes in tumor growth, metastasis, and cachexia. Malignant cells produce various soluble and cell surface signaling molecules to reprogram metabolic activity and production of growth factors/cytokines in adipocytes through endocrine, paracrine, and juxtacrine signaling mechanisms. After receiving signals from malignant cells, cancer-associated adipocytes (CAAs) produce various growth factors, adipokines, and adipocytokines that directly affect tumor cell growth and invasion. Alternatively, the adipocyte-derived factors have a significant impact on nontumor cells in the tumor microenvironment to modulate tumor growth, metastasis, and cachexia. The tumor cell-triggered metabolic reprograming in adipocytes releases metabolic products such as free fatty acids (FFAs) that will be used as energy fuel molecules to support tumor growth and metastasis. Lipolysis is also one of the key processes causing adipose atrophy and cachexia in cancer patients.

derived citrate is frequently used for lipogenesis and cholesterol synthesis $(58,59)$. The crucial lipogenic enzymes, including acetylCoA carboxylase, fatty acid synthase (FASN), and ATP citrate lyase, are ubiquitously expressed in most, if not all, cancer cells (2, $60,61)$. In particular, high levels of FASN are inversely correlated with survival prognosis (62). Some tumors, including PDAC and CRC, exhibit specific alterations in lipid metabolic pathways by generating high levels of signaling phosphatidylinositols, which serve as a predictive marker for prognosis $(62,63)$.

Cancer cells acquire exogenous FFAs released by CAAs through cell surface fatty acid translocase (also named CD36) (7, $64,65)$. Coculture of OC cells with omental adipocytes results in upregulation of $\mathrm{CD} 36$ expression, resulting in accelerated FFA uptake by cancer cells (66). Moreover, $\mathrm{CD}^{2} 6^{+}$cancer cells were shown to represent a metastasis-initiating cell subpopulation (7). FFAs provide an efficient source of energy production through the FAO pathway (3). Transport of acyl-CoAs into the mitochondria by carnitine palmitoyl transferase 1 (CPT1) protein is the rate-limiting step of FAO (67). However, metabolic stress often upregulates CPT1 in cancer cells, thereby enhancing FAO-mediated energy production $(67,68)$. In addition to mitochondria, $\mathrm{FAO}$ also occurs in peroxisomes within cancer cells (69). Targeting the FAO pathway, especially CPT1, provides an attractive approach for treating certain cancers. In fact, CPT1 inhibitors are under development for treating human cancers $(70,71)$.

\section{Impact of obesity on tumor growth and metastasis}

Epidemiological studies show a strong association between overweight and high incidence of a number of cancers, including endo- metrial, gastric, liver, kidney, esophageal, colorectal, pancreatic, gallbladder, breast, ovarian, prostate, and thyroid cancers, meningioma, and multiple myeloma (72). Obesity is also increasingly recognized as a poor prognostic marker for many cancers (73-75). Although the exact mechanism underlying obesity-driven high cancer risk and progression remains unknown, it is now acknowledged that the causal factors are complex. The following possible mechanisms have been suggested:

Phenotypic transition of adipocytes. After prolonged exposure to cancer cells, obese-subject-derived adipocytes manifest phenotypic changes, losing their lipid contents and gaining fibroblast/myofibroblast-like features to contribute to the cellular pool of CAFs $(14,15)$, which are known to promote cancer invasion and metastasis $(52,76,77)$.

Genomic instability. Obesity increases cellular oxidative stress and oxidative DNA damage, thus increasing cancer risks. For example, in mice expressing the prostate-specific Myc protein (ARR2/probasin-Myc or Hi-Myc mice), high-fat diet considerably increases prostate cancer incidence (78).

Low-grade inflammation. Obese adipose tissue is classified as a low-grade chronically inflammatory tissue, characterized by infiltration of a high number of macrophages, neutrophils, and other immune cells (79-81). Cancer-associated obese adipocytes (CAOAs) produce high levels of inflammatory cytokines, including IL-1 $\beta$, IL-6, IL-8, IL-10, and TNF- $\alpha$, to recruit and activate inflammatory cells (82). Moreover, TAMs are known to promote metastasis in multiple cancers (51).

Inhibition of apoptosis. Insulin is a potent cellular survival factor that induces proliferation and inhibits apoptosis in a broad range of cancer cells $(83,84)$. High insulin levels in obese individ- 
A

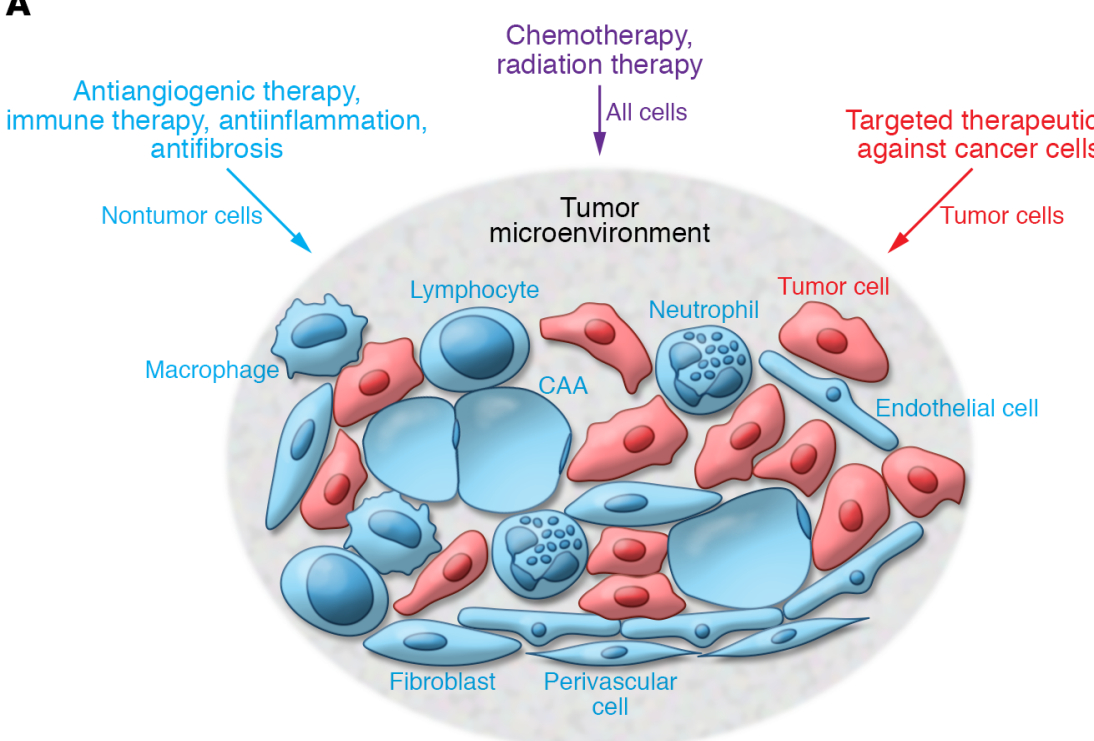

B

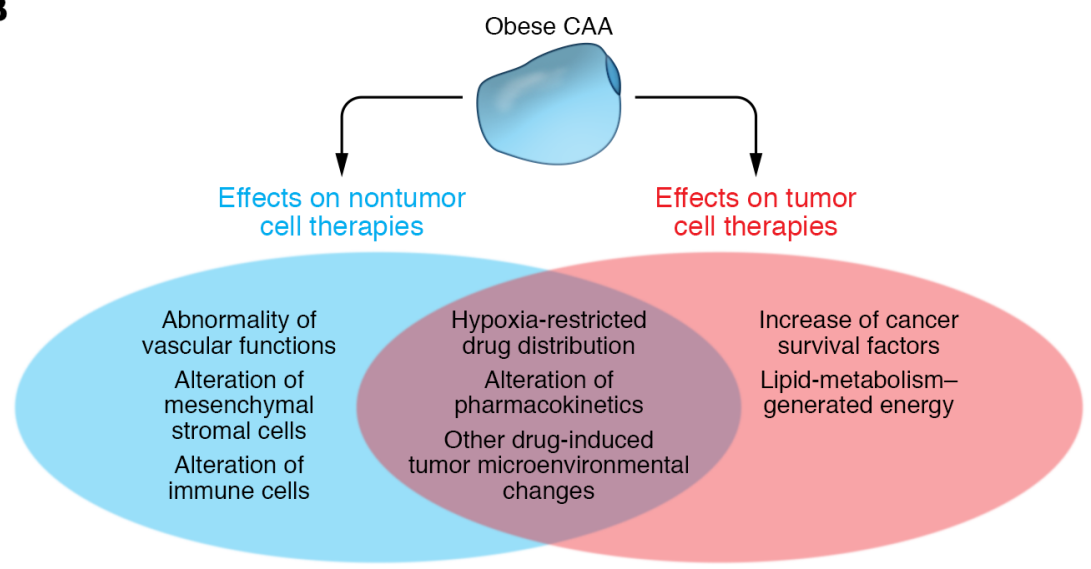

Figure 2. Mechanistic principles underlying cancer treatment and obesity-associated treatment resistance. (A) Mechanistic principles of cancer drugs. Conventional treatment approaches, including chemotherapeutics and radiation therapy, indistinguishably target malignant and nonmalignant cells in the tumor microenvironment and elsewhere in the body. Numerous targeted therapeutics targeting cancer cells have been developed, such as trastuzumab. Other targeted therapeutics, including antiangiogenic drugs, immune regulators, and antiinflammatory and antifibrotic drugs, aim to interfere with or enhance the interaction between nonmalignant cells and cancer cells. (B) Possible mechanisms of obese adipocytes in contributing to anticancer resistance. Obese adipocytes may have altered metabolism, pharmacokinetics, expression of tumor cell survival factors, immune cell functions, vascular functions, and drug distribution to affect anticancer drug responses. These alterations often lead to the development of resistance to cancer drugs.

uals and patients with type 2 diabetes mellitus are independently associated with several cancers, including CRC, BC, and PDAC $(85,86)$. Moreover, insulin also induces the expression of insulin growth factor-1 (IGF-1), which stimulates proliferation and survival of numerous cancers.

Angiogenesis. Obese WAT is highly vascularized, and continuous expansion of the adipose mass concomitantly accompanies neovascularization $(47,87,88)$. High density of preexisting and angiogenic microvessels in obese adipose tissues promotes tumorigenesis, malignant progression, and even metastasis (89). Alternatively, obese adipocytes located distally from the primary tumor release high levels of angiogenic cytokines and growth factors into the circulation, which switch on an angiogenic phenotype and initiate dormant tumor growth. This mechanism may explain why high-fat diet and obesity accelerate tumor growth without infiltrating into the tumor tissue.

Increase of circulating levels of fatty acidbinding proteins. Recent findings show that adipose fatty acid-binding protein (A-FABP) and FABP4 promote cancer development and metastasis, partly through the mechanism of TAM activation $(90,91)$. Circulating A-FABP is elevated in obese patients with breast cancer (92).

CAOAs may play dynamic roles in promoting tumor growth, metastasis, and drug responses. At the initial stage of tumor development, CAOA-derived abundant adipokines and adipocytokines facilitate tumor formation. Throughout tumor progression, excessive lipids stored in CAOAs provide metabolites and fuel for tumor expansion. At the advanced stage, depletion of lipid storage may switch CAOAs to become other cells in the TME such as CAFs, which promotes metastasis. Thus, the characteristic features of CAOAs are rather dynamic and unceasingly alter during cancer progression. Ultimately, these changes will result in alteration of cancer drug sensitivity.

\section{Targeting CAAs for drug development}

Targeted cancer therapeutics are classified into two categories (Figure 2A): drugs targeting cancer cells and drugs targeting cellular and molecular components of the TME.

Drugs targeting cancer cells. Cancer cells often overexpress and activate specific signaling molecules, and drugs that block these signaling pathways are commonly used to treat various cancers. For example, EGFR inhibitors and HER2-targeted drugs are successfully used in lung and breast cancer, respectively (93-95). Targeting tumor cell glycolysis is also an attractive approach for therapeutic intervention (96). However, development of effective therapeutics has been hampered by difficulty in defining cancer cell-specific targets that lack expression in normal cells. Several potential candidates, including glucose transporter 1 (GLUT1), hexokinases (HKs), phosphoglycerate dehydrogenase (PHGDH), and lactate dehydrogenase A (LDHA), are often overexpressed in many types of cancers (97). LDHA, which catalyzes the conversion of pyruvate to lactate, is a key checkpoint of anaerobic glycolysis and an attractive therapeutic target. Therapeutic efficacy of several small molecules exhibiting specific inhibitory activity against human LDH-5 (an isoform of LDH) has been validated in preclinical models $(98,99)$. However, none of these compounds has received approval for clinical uses. Similarly, ther- 
apeutics targeting GLUT1, HKs, PHGDH, and other glycolytic enzymes catalyzing intermediate steps are also at the preclinical stage of development owing to lack of cancer cell specificity (97).

Drugs targeting the TME's cellular and molecular components. Antiangiogenic drugs (AADs) targeting tumor blood vessels have become an important therapeutic modality for treating various cancers $(96,100,101)$. These drugs are often given to patients in combination with conventional chemotherapeutics (102-104). Another example is immunotherapy using the genetically propagated tumor antigen-recognizing lymphocytes (e.g., chimeric antigen receptor T [CAR T] cells) $(105,106)$, immune checkpoint inhibitors (107-109), and immunocytokines. Tumor inflammatory and fibrotic compartments are also attractive targets for treating various cancers. In fact, drugs targeting tumor inflammation and fibrosis are also successfully used for treating human cancers (110).

Nonmalignant cells, including adipocytes, constitute a substantial proportion of the tumor mass, and their contents are often correlated with a fast-growing and invasive phenotype. For example, the fibrotic component in some PDAC can occupy more than $90 \%$ of the entire tumor mass, and the degree of fibrosis is inversely correlated with the survival prognosis (111). As noncancer cells are common in the TME of all solid tumors, drugs targeting these cells are often used to treat various cancers and produce beneficial effects in human patients. For example, tumor growth is dependent on angiogenesis, and bevacizumab, a neutralizing antibody targeting VEGF, is widely used to treat various cancers in human patients (102). Drugs targeting TAMs, such as emactuzumab, a humanized anti-CSF1R neutralizing antibody, showed survival benefits in clinical trials for treating BC and OC (112). Celecoxib, an NSAID, shows clinical benefits for treating familial adenomatous polyposis and BC (113). Recently the FDA approved numerous immunotherapeutics, including checkpoint blockades such as antibodies blocking PD-1/PD-L1 and CTLA-4/B7-1/B7-2; CAR T cells; and immunocytokines. These drugs do not target cancer cells per se, but restore immune function in the TME to kill cancer cells. At the time of writing, unfortunately, drugs targeting CAAs are not available for treating human cancer patients.

Understanding the reciprocal interactions between cancer cells and adipocytes is crucial for developing effective cancer therapy. The following strategies should be considered when defining potential CAA therapeutic targets:

Blocking cancer cell-released signaling molecules. Cancer cells release signaling molecules that reprogram metabolic pathways in CAAs surrounding tumors and in distal locations. For example, BC cell-derived conditioned medium increases lipolysis in adipocytes, and the identity of these signaling molecules needs further investigation. Cancer cells produce cachexia factors including TNF- $\alpha$ and IL- 6 to trigger systemic lipolysis, resulting in adipose atrophy. Mechanisms underlying clinically available anticachexia drugs such as thalidomide and megestrol acetate are partly based on their effects of downregulating IL- 6 and TNF- $\alpha$ production $(114,115)$. However, clinical trials of specific blockades including infliximab (an anti-TNF- $\alpha$ neutralizing antibody) and etanercept (an anti-TNFR neutralizing antibody) showed no benefits against cachexia (52). By contrast, tocilizumab, an anti-IL-6 receptor antibody, demonstrated promising improvement of cachexia syndrome in a limited number of human patients (116).
Blocking CAA lipolysis. Inhibiting lipolysis would prevent release of FFA and other metabolites from CAAs and thus limit lipid fuel supply for cancer cells. At the time of writing, drugs based on this principle are not clinically available.

Blocking FFA uptake by cancer cells. Cancer cells express FFA receptors such as $\mathrm{CD} 36$ on their surface, and $\mathrm{CD} 36^{+}$cancer cells have been defined as a subpopulation of metastasis-initiating cells (7). Blocking of CD36 has demonstrated beneficial effects on suppression of tumor growth and metastasis in preclinical animal models.

Inhibiting CAA adipocytokine production. CAAs produce altered expression profiles of various cytokines that could markedly stimulate tumor cell growth and metastasis. Blocking CAAderived cytokines may be an important approach for cancer therapy.

Inhibiting CAA progenitor cell differentiation. In the TME, CAAs might be generated through a mechanism involving differentiation from mesenchymal stem cells. Blocking progenitor cell differentiation would potentially inhibit tumor growth and metastasis.

These therapeutic targets have not been validated in welldesigned clinical trials. Specific targeting of CAAs without an effect on other cells remains a challenging issue. Perhaps inhibition of CAA lipolysis would be the most attractive approach to limit FFA supply to tumor cells. Unraveling the mechanism that underlies tumor-triggered lipolysis would be beneficial for suppression of local tumor growth and global adipose atrophy. However, such approaches may also produce broad adverse effects and resistance to drugs. The history of experience with cancer therapy has taught us that monotherapy is generally less effective and often leads to resistance. Combination of various drugs with different mechanistic principles often improves therapeutic outcome by producing additive and sometimes synergistic effects. In this context, simultaneously targeting various CAA processes may be more beneficial if adverse effects are within a manageable level.

\section{Obese adipocytes confer chemoresistance}

Epidemiological evidence recognizes worse clinical outcomes in obese cancer patients compared with nonobese patients, despite the fact that they receive the same therapeutic agents (117-119). Clinical experiences demonstrate that the dose intensity of chemotherapeutics correlates with therapeutic benefits and toxicity $(120,121)$. Most studies choose BC to correlate obesity and anticancer drug responses $(19,82,119)$ because the most common obesity-associated cancer types are breast, endometrial, and ovarian cancers in postmenopausal women (72). Additionally, the proximity of $\mathrm{BC}$ to adipose tissue, standardized therapeutic regimens, and elimination of the gender issue have made it easier to choose this cancer type to study the relation between obesity and anticancer drug response. Several possible mechanisms have been proposed to explain the poor clinical outcomes observed in obese cancer patients (Figure 2B).

Presence of more advanced disease in obese cancer patients at the time of diagnosis. Advanced tumors are difficult to treat and are often resistant to therapeutics. Experimental data show that BC, $\mathrm{OC}$, and $\mathrm{PC}$ in obese adipose tissues grow faster and become more invasive $(22,122,123)$.

Adipose hypoxia. Obese adipose tissue may experience insufficient blood perfusion owing to a relatively low density of microvessels. The enlarged size of adipocytes in obese subjects increases the 
intercapillary distance that would limit the oxygen perfusion (124, 125). Along this line of thought, it has been reported that blood flow is approximately $30 \%-40 \%$ lower in obese adipose tissues versus their lean counterparts (126). Thus, obese adipose tissues experience low-grade hypoxia, affecting the cellular and molecular compositions in the adipose microenvironment. For example, hypoxia triggers angiogenesis, inhibits macrophage migration, inhibits preadipocyte differentiation, augments fibrosis, and suppresses recruitment of immune cells $(127,128)$. These alterations would inevitably affect drug responses of a tumor growing in the neighborhood of obese adipose tissue. Adipose hypoxia is likely to constrain anticancer drug distribution within a tumor grown in the obese adipose tissue. Even though the tumor tissue is hypervascularized, the disorganized, tortuous, and leaky tumor vasculature is poorly perfused, and drug perfusion is limited $(129,130)$. Our recent findings show that the hypoxic obese adipose tissue would further elevate tumor hypoxia $(3,131)$. In obese adipose tissue, the enlarged adipocyte size increases the intercellular distance, creating a relatively hypoxic environment (132). Growing a tumor in the hypoxic obese adipose tissue may further boost hypoxia in tumor tissues owing to hypovascularity and poor vascular perfusion.

Alteration of pharmacokinetics. Only very limited data on the impact of obesity on the pharmacokinetics of chemotherapeutics are available (133-135). For lipophilic drugs, volume distribution seems to increase in obese patients relative to nonobese patients. As the liver is the primary organ for drug clearance, blood perfusion and steatosis could affect drug clearance. Obese subjects often have steatotic livers that might alter drug clearance (136). Drug clearance in the kidney might also be altered in obese cancer patients as a result of changes of the glomerular filtration rate (137).

Influential effects of other drugs in combination therapy settings. Other therapeutics are often administered to cancer patients in combination with chemotherapeutics. It has been shown that bevacizumab and other VEGF inhibitors alter distribution of chemotherapeutics in tumors (138). In preclinical animal models, anti-VEGF treatment causes a higher degree of tumor hypoxia in obese animals compared with lean animals $(3,19)$. Severe hypoxia would be expected to further restrain tissue distribution of chemotherapeutics.

Alterations of metabolic program. Increasing evidence indicates that intracellular ATP level is a critical factor for predicting chemoresistance (139-141). Hypoxia triggers lipid metabolism in adipocytes and cancer cells to produce high levels of ATP, contributing to chemoresistance (140).

Production of tumor-promoting growth factors and cytokines. Obese adipocytes often produce high levels of growth factors and cytokines that improve cancer cell survival and resistance to chemotherapy (133). For example, high levels of leptin production in obese subjects could protect cancer cells from chemotherapyinduced apoptosis (142).

Enhancement of cancer fibrosis. It has been suggested that obesity may augment the fibrotic reaction in tumors by promoting proinflammation and fibrosis crosstalk, diminishing drug delivery in tumors (143).

Alteration of microbiota. High-fat diet and obesity often cause dysbiosis and microbial imbalance that contribute to chemoresistance. For example, increased Fusobacterium nucleatum in CRC patients contributes to high recurrence after chemotherapy (144).
Generation of drug-resistant cancer stem cells. Obese adipocytes upregulate expression levels of the FFA translocase CD36 and drive cancer cells to gain stemness features (66). Increased FFA uptake by elevated CD36 would reprogram metabolism and drive cell proliferation and metastasis. Indeed, CD36 also defines a metastasisinitiating cell subpopulation (7). Cancer stem cells acquire chemoresistance through several mechanisms, including avoidance of drug exposure, avoidance of molecular targets for drugs, intracellular inactivation of drugs, remaining dormancy, repair of DNA damage, enhancement of survival signals, and self-regeneration (145).

Adipocyte-derived conditioned medium protects BC and PDAC cells from the cytotoxic effects of gemcitabine chemotherapy $(146,147)$. Similarly, culture media from adipocytes compromise melanoma cell chemosensitivity to cisplatin and docetaxel (148). Adipocyte-derived leptin is defined as the key survival factor for counteracting the chemosensitivity $(149,150)$. Along this line, adipocyte-derived leptin also contributes to chemoresistance to 5-fluorouracil in pancreatic and colorectal cancers $(142,150)$. Another study shows that CAA-derived microRNA-21-containing exosomes confer chemoresistance of OC by targeting apoptotic protease-activating factor 1 (APAF1) (151).

In addition to solid cancers, adipocytes also substantially contribute to chemoresistance of various leukemic cells (152-157). A substantial portion of the BM stroma is composed of adipocytes that are in close interaction with cancer cells in patients with leukemia. Obesity is inversely correlated with event-free survival of hematological malignancies, including acute lymphoblastic leukemia (ALL) and lymphoma (158-160). Obese adipocytes protect ALL from a variety of chemotherapeutic agents $(152,154,155)$. It has been suggested that leukemic cells trigger oxidative stress in adipocytes, which in turn produce survival factors that protect leukemic cells from chemotherapy (154). In myeloma, mature BM adipocytes protect cancer cells from chemotherapy by activating autophagy and suppressing apoptosis (157).

In summary, multifarious and complex mechanisms are involved in adipocyte- and lipid metabolism-mediated chemoresistance in various solid and nonsolid cancers. Targeting CAAs may have a profound beneficial impact on the efficacy of chemotherapeutics.

\section{CAAs and lipid metabolism in antiangiogenic drug resistance}

Cancer drugs targeting tumor blood vessels are frequently used for treating various cancers, and clinical benefits remain modest (161-163). Intrinsic and acquired AAD resistance often occurs in cancer patients and considerably hampers therapeutic efficacy $(164,165)$. Recent work from our laboratory demonstrates that genetically identical tumors implanted in different locations exhibit differential responses to AAD treatment, demonstrating a crucial role of the extratumoral tissue environment in contributing to AAD resistance (3). For example, implantation of a $\mathrm{CRC}$ or PDAC tumor in adipose tissue resulted in intrinsic AAD resistance, whereas the same tumor grown in nonadipose tissue is sensitive to identical AAD treatment (3). Likewise, an AADsensitive hepatocellular carcinoma (HCC) grown in steatotic livers acquires resistance to antiangiogenic therapy, whereas HCCs in nonsteatotic livers remain sensitive. These preclinical findings link AAD resistance to the adipose tissue environment. Surpris- 


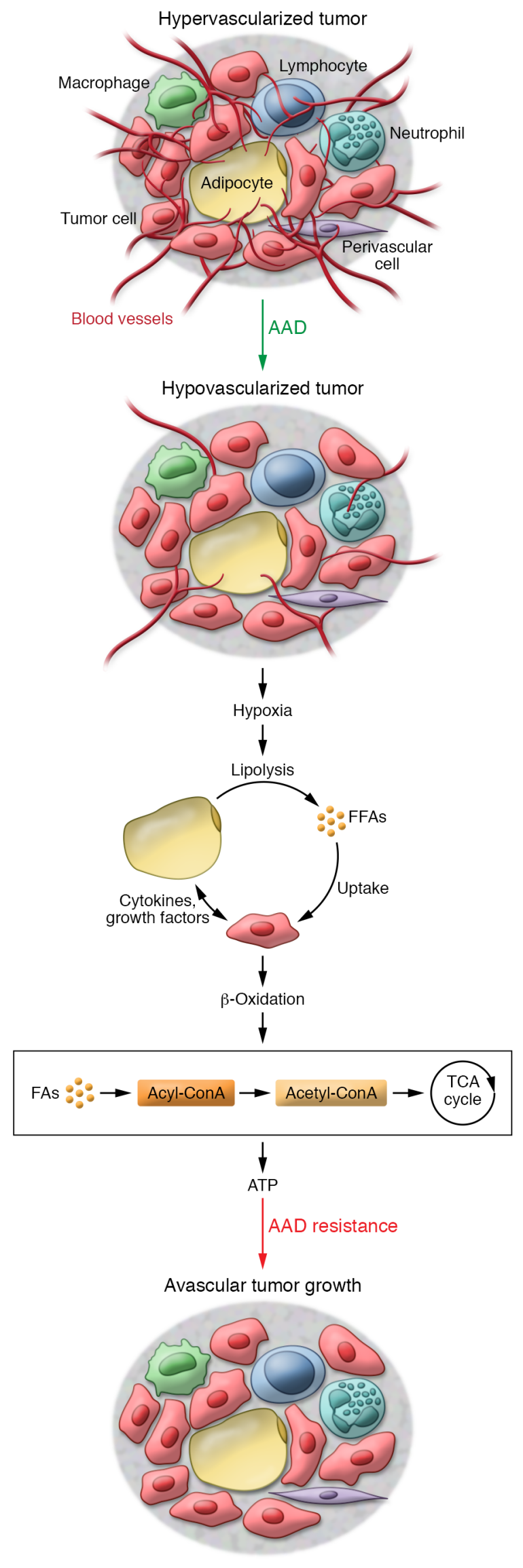

Figure 3. The mechanism of adipocyte metabolic reprogramming in antiangiogenic drug resistance. Treatment of tumors with antiangiogenic drugs reduces the tumor vascular density, leading to tissue hypoxia. Hypoxia triggers lipolysis in tumor-infiltrating and peritumoral CAAs to produce excessive FFAs. Hypoxia also upregulates the expression levels of CD36, the fatty acid translocase or receptor, in cancer cells to increase FFA uptake. Within tumor cells, FFA through the $\beta$-oxidation pathway is metabolized to produce ATP that supports tumor cell proliferation and migration in the presence of a minimal number of microvessels. This mechanism explains in part how CAAs contribute to antiangiogenic drug resistance.

ingly, microvessels in nonadipose and adipose tumors are equally sensitive in response to AAD treatment (3). However, tumors in the adipose environment continue to grow into large masses in the presence of a minimal number of intratumoral microvessels. According to the established dogma, tumor growth is dependent on active angiogenesis, and inhibition of angiogenesis would attenuate tumor growth (101). Indeed, vascular reduction matches tumor inhibition in nonadipose tissues $(166,167)$. Thus, the unexpected finding from tumors grown in adipose tissue differentiates the antiangiogenic response from tumor growth.

How could a small number of microvessels support continuous expansion of a growing tumor tissue? In response to anti-VEGF treatment, tumors grown in the adipose environment experience more severe hypoxia than those in nonadipose tissues (Figure 3). It appears that adipose and hepatic vasculatures are dependent on VEGF, and blocking VEGF produces marked vascular regression $(88,145)$. Tumor vessels originating from these tissues may genetically carry their intrinsic features, including VEGF-dependent survival. AAD-induced vascular reduction and hypoxia would confine the supply of circulating glucose, jeopardizing glucosedependent metabolism, i.e., the Warburg effect for energy production $(1,54,168)$. To survive and proliferate, cancer cells must use alternative mechanisms for energy production.

AAD-triggered hypoxia instigates three processes of lipid metabolism (ref. 3 and Figure 3): (a) hypoxia promotes lipolysis in adipocytes, which release glycerol and FFAs as final metabolites; (b) cancer cells increase FFA uptake by upregulating CD36; and (c) cancer cells undergo reprogramming of metabolic pathways, activating the $\beta$-oxidation pathway to produce FFA metabolismdependent energy production that supports continuous tumor growth and even metastasis $(25,169,170)$. Based on these findings, it would be reasonable to design a strategy that overcomes $\mathrm{AAD}$ resistance by combining antiangiogenic therapy with lipid metabolism inhibitors. In support of this view, combination of AAD with a $\beta$-oxidation inhibitor such as the CPT1 inhibitor etomoxir produces greater anticancer effects in an animal model of HCC grown in steatotic liver (3).

Clinical studies show an inverse correlation between obesity and clinical benefits in patients who receive antiangiogenic therapy (171-174). In human BC patients, anti-VEGF treatment induces high expression levels of IL-6 and FGF2, which contribute to AAD resistance. Again, anti-VEGF-induced hypoxia triggers the high expression of IL-6 and FGF2 (19). Furthermore, the number of adipocytes within a tumor directly correlates with poor AAD response. In addition to obese adipocytes, tumor-infiltrating inflammatory cells also contribute to high production of angiogenic cytokines that are not targets of AADs (19, 175-177). 


\section{CAAs in radiotherapy and targeted drug resistance}

The efficacy of radiotherapy is largely dependent on the availability of oxygen molecules that react with water radiolysis to release DNA-damaging reactive oxygen species (ROS) (178-180). High adiposity and obesity are considered chronic low-grade inflammation with enduring and increased oxidative stress $(181,182)$. Several obese adipocyte-derived adipokines and adipocytokines, including leptin, adiponectin, TNF- $\alpha$, and IL- 6 , induce the production of ROS to generate oxidative stress (183-185). The high adiposity-linked oxidative stress reduces availability of free oxygen molecules and contributes to radioresistance. As previously discussed, obese adipose tissue generally experiences tissue hypoxia (127, 186-189), and recruitment of obese adipocytes in tumor tissues further facilitates tumor hypoxia $(3,19,124)$, thereby leading to resistance to radiation therapy.

Mature adipocytes improve $\mathrm{BC}$ cell survival by producing soluble factors that activate the Chk1-mediated pathway (13). It appears that adipocyte-derived factors stimulate malignant cells to produce high levels of IL-6, which protects cancer cells from radiation therapy (13). Another study shows that melanoma cells gain radioresistance after exposure to adipocyte secretome through a protective mechanism involving oxidative stress and cell survival $(13,190,191)$. At the molecular level, activation of the AKT pathway in cancer cells by adipocyte-secreted factors is involved in development of radiotherapy resistance (190). Independent evidence from other studies further supports the causal link between obesity and radioresistance. For example, obesity contributes to radiotherapy resistance by enhancing genetic instability of esophageal adenocarcinoma (192-194). Obese adipocyte stem cells promote estrogen receptor-positive $\left(\mathrm{ER}^{+}\right) \mathrm{BC}$ cell survival after exposure to radiation (195).

In addition to radioresistance, adipocytes also confer drug resistance to targeted therapeutics. A large clinical study of HER2 ${ }^{+}$BC patients showed that obese patients have worse outcomes than normal-weight patients (196-198). A potential association between obesity and trastuzumab resistance was established based on clinical and preclinical studies: several studies demonstrated that adipocytes and preadipocytes compromise trastuzumab and tamoxifen sensitivity by producing high levels of tumor cell survival factors, inhibition of IFN- $\gamma$ production in natural killer cells, and high expression of inflammatory cytokines and adipokines, including IL- 6 , TNF- $\alpha$, and leptin $(133,199,200)$.

\section{Conclusions and future directions}

The role of adipocytes in cancer drug resistance is often overlooked. We are beginning to understand the complex roles of adipocytes and lipid metabolism in modulation of anticancer drug sensitivity. CAAs located within the tumor tissues, in peritumoral regions, and even in distal tissues substantially affect tumor growth, metastasis, and drug sensitivity through multifarious mechanisms, including paracrine, juxtacrine, and endocrine signaling, metabolites, and metabolic reprogramming. Thus, targeting CAAs and lipid metabolism would provide an attractive approach for cancer therapy. However, targeting CAAs alone for cancer therapy is less likely to be effective, because this approach may not eliminate malignant cells. Based on preclinical findings, combination therapy consisting of cytostatic agents, targeted therapeutics, and lipid metabolism inhibitors would be reasonably more effective for treating cancers.

Cancer is a systemic disease that often causes adipose atrophy and cancer cachexia owing to metabolic dysfunction. In fact, cachexia is responsible for about $25 \%$ of mortality of all cancer patients, and unfortunately no effective treatment is available despite the frantic efforts for cancer drug development. Targeting of the lipolysis pathway may be beneficial in cancer therapy through several mechanisms, including (a) limiting the supply of FFA to tumor cells to reduce their source of energy production; (b) preventing adipose atrophy and cachexia development; and (c) sensitizing to drugs targeting cancer cells and angiogenesis. Based on these mechanisms, future development of new therapeutics targeting lipid metabolism would offer exciting opportunities for cancer therapy.

\section{Acknowledgments}

YC's laboratory is supported through research grants from the European Research Council (advanced grant ANGIOFAT, project 250021), the Swedish Research Council, the Swedish Cancer Foundation, the Swedish Children's Cancer Foundation, and the Karolinska Institute Foundation; the Karolinska Institute Distinguished Professor Award; the Strategic Research Areas of Karolinska Institutet; the Torsten Soderbergs Foundation; the Maud and Birger Gustavsson Foundation; the Novo Nordisk FoundationAdvance grant; and the Knut and Alice Wallenberg Foundation.

Address correspondence to: Yihai Cao, Department of Microbiology, Tumor and Cell Biology, Karolinska Institutet, Biomedicum, Solnavägen 9, 17175, Stockholm, Sweden. Phone: 46.8.5248.7596; Email:yihai.cao@ki.se.
1. Vander Heiden MG, Cantley LC, Thompson CB. Understanding the Warburg effect: the metabolic requirements of cell proliferation. Science. 2009;324(5930):1029-1033.

2. Beloribi-Djefaflia S, Vasseur S, Guillaumond F. Lipid metabolic reprogramming in cancer cells. Oncogenesis. 2016;5:e189.

3. Iwamoto H, et al. Cancer lipid metabolism confers antiangiogenic drug resistance. Cell Metab. 2018;28(1):104-117.e5.

4. Liu Y, Zuckier LS, Ghesani NV. Dominant uptake of fatty acid over glucose by prostate cells: a potential new diagnostic and therapeutic approach. Anticancer Res. 2010;30(2):369-374.

5. Caro P, et al. Metabolic signatures uncover distinct targets in molecular subsets of diffuse large B cell lymphoma. Cancer Cell. 2012;22(4):547-560.

6. Li J, Cheng JX. Direct visualization of de novo lipogenesis in single living cells. Sci Rep. 2014;4:6807.

7. Pascual G, et al. Targeting metastasis-initiating cells through the fatty acid receptor CD36. Nature. 2017;541(7635):41-45.

8. Yue S, et al. Cholesteryl ester accumulation induced by PTEN loss and PI3K/AKT activation underlies human prostate cancer aggressive- ness. Cell Metab. 2014;19(3):393-406.

9. Demark-Wahnefried W, et al. The role of obesity in cancer survival and recurrence. Cancer Epidemiol Biomarkers Prev. 2012;21(8):1244-1259.

10. Protani M, Coory M, Martin JH. Effect of obesity on survival of women with breast cancer: systematic review and meta-analysis. Breast Cancer Res Treat. 2010;123(3):627-635.

11. Sinicrope FA, Foster NR, Sargent DJ, O'Connell MJ, Rankin C. Obesity is an independent prognostic variable in colon cancer survivors. Clin Cancer Res. 2010;16(6):1884-1893.

12. Balaban S, et al. Adipocyte lipolysis links obesity 
to breast cancer growth: adipocyte-derived fatty acids drive breast cancer cell proliferation and migration. Cancer Metab. 2017;5:1.

13. Bochet L, Meulle A, Imbert S, Salles B, Valet P, Muller C. Cancer-associated adipocytes promotes breast tumor radioresistance. Biochem Biophys Res Commun. 2011;411(1):102-106.

14. Dirat B, et al. Cancer-associated adipocytes exhibit an activated phenotype and contribute to breast cancer invasion. Cancer Res. 2011;71(7):2455-2465.

15. Bochet L, et al. Adipocyte-derived fibroblasts promote tumor progression and contribute to the desmoplastic reaction in breast cancer. Cancer Res. 2013;73(18):5657-5668.

16. Muller C. Tumour-surrounding adipocytes are active players in breast cancer progression. Ann Endocrinol (Paris). 2013;74(2):108-110.

17. Argilés JM, Stemmler B, López-Soriano FJ, Busquets S. Inter-tissue communication in cancer cachexia. Nat Rev Endocrinol. 2018;15(1):9-20.

18. Morris EV, Edwards CM. The role of bone marrow adipocytes in bone metastasis. JBone Oncol. 2016;5(3):121-123.

19. Incio J, et al. Obesity promotes resistance to antiVEGF therapy in breast cancer by up-regulating IL-6 and potentially FGF-2. Sci Transl Med. 2018;10(432):eaag0945.

20. Nieman KM, Romero IL, Van Houten B, Lengyel E. Adipose tissue and adipocytes support tumorigenesis and metastasis. Biochim Biophys Acta. 2013;1831(10):1533-1541.

21. Khalid A, et al. Recent advances in discovering the role of CCL 5 in metastatic breast cancer. Mini Rev Med Chem. 2015;15(13):1063-1072.

22. Laurent $\mathrm{V}$, et al. Periprostatic adipocytes act as a driving force for prostate cancer progression in obesity. Nat Commun. 2016;7:10230.

23. Argilés JM, Busquets S, Stemmler B, LópezSoriano FJ. Cancer cachexia: understanding the molecular basis. Nat Rev Cancer. 2014;14(11):754-762.

24. Petruzzelli M, et al. A switch from white to brown fat increases energy expenditure in cancer-associated cachexia. Cell Metab. 2014;20(3):433-447.

25. Nieman KM, et al. Adipocytes promote ovarian cancer metastasis and provide energy for rapid tumor growth. Nat Med. 2011;17(11):1498-1503.

26. Huang CK, et al. Adipocytes promote malignant growth of breast tumours with monocarboxylate transporter 2 expression via $\beta$-hydroxybutyrate. Nat Commun. 2017;8:14706.

27. Xiong Y, Russell DL, McDonald LT, Cowart LA, LaRue AC. Hematopoietic stem cell-derived adipocytes promote tumor growth and cancer cell migration. Int J Cancer Res Mol Mech. 2017;3(1):10.16966/2381-3318.130.

28. Yang D, et al. Utilization of adipocyte-derived lipids and enhanced intracellular trafficking of fatty acids contribute to breast cancer progression. Cell Commun Signal. 2018;16(1):32.

29. Zhang M, et al. Adipocyte-derived lipids mediate melanoma progression via FATP proteins. Cancer Discov. 2018;8(8):1006-1025.

30. Ray A, Cleary MP. The potential role of leptin in tumor invasion and metastasis. Cytokine Growth Factor Rev. 2017;38:80-97.

31. Wang $\mathrm{CH}$, et al. Resistin facilitates breast cancer progression via TLR4-mediated induction of mesenchymal phenotypes and stemness properties. Oncogene. 2018;37(5):589-600.

32. Goto $\mathrm{H}$, et al. Adipose-derived stem cells enhance human breast cancer growth and cancer stem cell-like properties through adipsin. Oncogene. 2019;38(6):767-779.

33. Lin $\mathrm{T}$. The role of visfatin in cancer proliferation, angiogenesis, metastasis, drug resistance and clinical prognosis. Cancer Manag Res. 2019;11:3481-3491.

34. Lu Z, et al. The serum biomarker chemerin promotes tumorigenesis and metastasis in oral squamous cell carcinoma. Clin Sci. 2019;133(5):681-695.

35. Yu H, Lee H, Herrmann A, Buettner R, Jove R. Revisiting STAT3 signalling in cancer: new and unexpected biological functions. Nat Rev Cancer. 2014;14(11):736-746.

36. Elaraj DM, et al. The role of interleukin 1 in growth and metastasis of human cancer xenografts. Clin Cancer Res. 2006;12(4):1088-1096.

37. Balkwill $F$. Tumour necrosis factor and cancer. Nat Rev Cancer. 2009;9(5):361-371.

38. Qian BZ, et al. CCL2 recruits inflammatory monocytes to facilitate breast-tumour metastasis. Nature. 2011;475(7355):222-225.

39. Kim KJ, et al. Inhibition of vascular endothelial growth factor-induced angiogenesis suppresses tumour growth in vivo. Nature. 1993;362(6423):841-844.

40. Jiramongkolchai P, Owens P, Hong CC. Emerging roles of the bone morphogenetic protein pathway in cancer: potential therapeutic target for kinase inhibition. Biochem Soc Trans. 2016;44(4):1117-1134.

41. Stein U, et al. MACC1, a newly identified key regulator of HGF-MET signaling, predicts colon cancer metastasis. Nat Med. 2009;15(1):59-67.

42. Pollak M. The insulin and insulin-like growth factor receptor family in neoplasia: an update. Nat Rev Cancer. 2012;12(3):159-169.

43. Pickup M, Novitskiy S, Moses HL. The roles of TGF $\beta$ in the tumour microenvironment. Nat Rev Cancer. 2013;13(11):788-799.

44. Kessenbrock K, Plaks V, Werb Z. Matrix metalloproteinases: regulators of the tumor microenvironment. Cell. 2010;141(1):52-67.

45. Xiang F, et al. Omental adipocytes enhance the invasiveness of gastric cancer cells by oleic acid-induced activation of the PI3K-Akt signaling pathway. Int J Biochem Cell Biol. 2017;84:14-21.

46. Motrescu ER, Rio MC. Cancer cells, adipocytes and matrix metalloproteinase 11: a vicious tumor progression cycle. Biol Chem. 2008;389(8):1037-1041.

47. Cao Y. Angiogenesis modulates adipogenesis and obesity. J Clin Invest. 2007;117(9):2362-2368.

48. Chaurasia B, et al. Adipocyte ceramides regulate subcutaneous adipose browning, inflammation, and metabolism. Cell Metab. 2016;24(6):820-834.

49. Arcidiacono B, et al. Expression of matrix metalloproteinase-11 is increased under conditions of insulin resistance. World J Diabetes. 2017;8(9):422-428.

50. Tan J, et al. Stromal matrix metalloproteinase-11 is involved in the mammary gland postnatal development. Oncogene. 2014;33(31):4050-4059.

51. Pollard JW. Tumour-educated macrophages promote tumour progression and metastasis. Nat Rev Cancer. 2004;4(1):71-78.

52. Yang $\mathrm{Y}$, et al. The PDGF-BB-SOX7 axis-modulated IL-33 in pericytes and stromal cells promotes metastasis through tumour-associated macrophages. Nat Commun. 2016;7:11385.

53. Viña J, et al. Mitochondrial biogenesis in exercise and in ageing. Adv Drug Deliv Rev . 2009;61(14):1369-1374.

54. Koppenol WH, Bounds PL, Dang CV. Otto Warburg's contributions to current concepts of cancer metabolism. Nat Rev Cancer. 2011;11(5):325-337.

55. Warburg O. On the origin of cancer cells. Science. 1956;123(3191):309-314.

56. Bui T, Thompson CB. Cancer's sweet tooth. Cancer Cell. 2006;9(6):419-420.

57. Petan T, Jarc E, Jusović M. Lipid droplets in cancer: guardians of fat in a stressful world. Molecules. 2018;23(8):1941.

58. Koo JH, Guan KL. Interplay between YAP/TAZ and metabolism. Cell Metab. 2018;28(2):196-206.

59. Israël M, Schwartz L. The metabolic advantage of tumor cells. Mol Cancer. 2011;10:70.

60. Menendez JA, Lupu R. Fatty acid synthase and the lipogenic phenotype in cancer pathogenesis. Nat Rev Cancer. 2007;7(10):763-777.

61. Zaidi N, Swinnen JV, Smans K. ATP-citrate lyase: a key player in cancer metabolism. Cancer Res. 2012;72(15):3709-3714.

62. Kuhajda FP. Fatty acid synthase and cancer: new application of an old pathway. Cancer Res. 2006;66(12):5977-5980.

63. Vargas T, et al. ColoLipidGene: signature of lipid metabolism-related genes to predict prognosis in stage-II colon cancer patients. Oncotarget. 2015;6(9):7348-7363.

64. Hoebe $\mathrm{K}$, et al. CD36 is a sensor of diacylglycerides. Nature. 2005;433(7025):523-527.

65. Jaqaman K, et al. Cytoskeletal control of CD36 diffusion promotes its receptor and signaling function. Cell. 2011;146(4):593-606.

66. Ladanyi A, et al. Adipocyte-induced CD36 expression drives ovarian cancer progression and metastasis. Oncogene. 2018;37(17):2285-2301.

67. Zaugg K, et al. Carnitine palmitoyltransferase $1 \mathrm{C}$ promotes cell survival and tumor growth under conditions of metabolic stress. Genes Dev. 2011;25(10):1041-1051.

68. Reilly PT, Mak TW. Molecular pathways: tumor cells co-opt the brain-specific metabolism gene CPT1C to promote survival. Clin Cancer Res. 2012;18(21):5850-5855.

69. Poirier Y, Antonenkov VD, Glumoff T, Hiltunen JK. Peroxisomal beta-oxidation-a metabolic pathway with multiple functions. Biochim Biophys Acta. 2006;1763(12):1413-1426.

70. Carracedo A, Cantley LC, Pandolfi PP. Cancer metabolism: fatty acid oxidation in the limelight. Nat Rev Cancer. 2013;13(4):227-232.

71. Crunkhorn S. Breast cancer: inhibiting fatty acid oxidation blocks tumour growth. Nat Rev Drug Discov. 2016;15(5):310.

72. De Pergola G, Silvestris F. Obesity as a major risk factor for cancer. JObes. 2013;2013:291546.

73. Parekh N, Chandran U, Bandera EV. Obesity in cancer survival. Annu Rev Nutr. 2012;32:311-342.

74. Carmichael AR. Obesity as a risk factor for development and poor prognosis of breast cancer. 
BJOG. 2006;113(10):1160-1166.

75. Martinez-Useros J, Garcia-Foncillas J. Obesity and colorectal cancer: molecular features of adipose tissue. J Transl Med. 2016;14:21.

76. Andersson P, et al. Molecular mechanisms of IL-33-mediated stromal interactions in cancer metastasis. JCI Insight. 2018;3(20):122375.

77. Liu C, et al. A zebrafish model discovers a novel mechanism of stromal fibroblastmediated cancer metastasis. Clin Cancer Res. 2017;23(16):4769-4779.

78. Kobayashi N, et al. Effect of low-fat diet on development of prostate cancer and Akt phosphorylation in the Hi-Myc transgenic mouse model. Cancer Res. 2008;68(8):3066-3073.

79. Heilbronn LK, Campbell LV. Adipose tissue macrophages, low grade inflammation and insulin resistance in human obesity. Curr Pharm Des. 2008;14(12):1225-1230.

80. Riondino S, Roselli M, Palmirotta R, DellaMorte D, Ferroni P, Guadagni F. Obesity and colorectal cancer: role of adipokines in tumor initiation and progression. World J Gastroenterol. 2014;20(18):5177-5190.

81. Saltiel AR, Olefsky JM. Inflammatory mechanisms linking obesity and metabolic disease. J Clin Invest. 2017;127(1):1-4.

82. Duong MN, Geneste A, Fallone F, Li X, Dumontet $\mathrm{C}$, Muller $\mathrm{C}$. The fat and the bad: mature adipocytes, key actors in tumor progression and resistance. Oncotarget. 2017;8(34):57622-57641.

83. Christofori G, Naik P, Hanahan D. A second signal supplied by insulin-like growth factor II in oncogene-induced tumorigenesis. Nature. 1994;369(6479):414-418.

84. Palanichamy JK, et al. RNA-binding protein IGF2BP3 targeting of oncogenic transcripts promotes hematopoietic progenitor proliferation. JClin Invest. 2016;126(4):1495-1511.

85. Mendonça FM, et al. Metabolic syndrome and risk of cancer: which link? Metab Clin Exp. 2015;64(2):182-189.

86. Zhang D, Zhao Y, Wang T, Xi Y, Li N, Huang H. Diabetes mellitus and long-term mortality of ovarian cancer patients. A systematic review and meta-analysis of 12 cohort studies. Diabetes Metab Res Rev. 2017;33(4):10.1002/dmrr.2868.

87. Bråkenhielm E, et al. Angiogenesis inhibitor, TNP-470, prevents diet-induced and genetic obesity in mice. Circ Res. 2004;94(12):1579-1588.

88. Honek J, et al. Modulation of age-related insulin sensitivity by VEGF-dependent vascular plasticity in adipose tissues. Proc Natl Acad Sci U S A. 2014;111(41):14906-14911.

89. Lim S, Hosaka K, Nakamura M, Cao Y. Co-option of pre-existing vascular beds in adipose tissue controls tumor growth rates and angiogenesis. Oncotarget. 2016;7(25):38282-38291.

90. Gharpure KM, et al. FABP 4 as a key determinant of metastatic potential of ovarian cancer. Nat Commun. 2018;9(1):2923.

91. Hao J, et al. Expression of adipocyte/macrophage fatty acid-binding protein in tumor-associated macrophages promotes breast cancer progression. Cancer Res. 2018;78(9):2343-2355.

92. Hao J, et al. Circulating adipose fatty acid binding protein is a new link underlying obesity-associated breast/mammary tumor development. Cell
Metab. 2018;28(5):689-705.e5.

93. Choi JY, Choi YJ, Shin SW. Osimertinib in EGFR mutation-positive advanced NSCLC. $N$ EnglJ Med. 2018;378(13):1262.

94. Rich JN, Rasheed BK, Yan H. EGFR mutations and sensitivity to gefitinib. N Engl J Med. 2004;351(12):1260-1261.

95. Slamon D, et al. Adjuvant trastuzumab in HER2-positive breast cancer. $N$ Engl J Med. 2011;365(14):1273-1283.

96. Tennant DA, Durán RV, Gottlieb E. Targeting metabolic transformation for cancer therapy. Nat Rev Cancer. 2010;10(4):267-277.

97. Hamanaka RB, Chandel NS. Targeting glucose metabolism for cancer therapy. JExp Med. 2012;209(2):211-215.

98. Rani R, Kumar V. Recent update on human lactate dehydrogenase enzyme 5 (hLDH5) inhibitors: a promising approach for cancer chemotherapy. J Med Chem. 2016;59(2):487-496.

99. Granchi C, Paterni I, Rani R, Minutolo F. Small-molecule inhibitors of human LDH5. Future Med Chem. 2013;5(16):1967-1991.

100.Cao Y, et al. Forty-year journey of angiogenesis translational research. Sci Transl Med. 2011;3(114):114rv3.

101.Folkman J. Tumor angiogenesis: therapeutic implications. N Engl J Med. 1971;285(21):1182-1186.

102. Hurwitz $\mathrm{H}$, et al. Bevacizumab plus irinotecan, fluorouracil, and leucovorin for metastatic colorectal cancer. NEnglJ Med. 2004;350(23):2335-2342.

103. Miller K, et al. Paclitaxel plus bevacizumab versus paclitaxel alone for metastatic breast cancer. N Engl J Med. 2007;357(26):2666-2676.

104.Ueda S, Saeki T, Osaki A, Yamane T, Kuji I. Bevacizumab induces acute hypoxia and cancer progression in patients with refractory breast cancer: multimodal functional imaging and multiplex cytokine analysis. Clin Cancer Res. 2017;23(19):5769-5778.

105. Quintás-Cardama A. CAR T-cell therapy in large B-cell lymphoma. $N$ Engl J Med. 2018;378(11):1065.

106.Abramson JS, et al. Anti-CD19 CAR T cells in CNS diffuse large-B-cell lymphoma. $N$ Engl $J$ Med. 2017;377(8):783-784.

107. Brahmer JR, et al. Safety and activity of antiPD-L1 antibody in patients with advanced cancer. N Engl JMed. 2012;366(26):2455-2465.

108. Ribas A. Tumor immunotherapy directed at PD-1. N EnglJMed. 2012;366(26):2517-2519.

109. Robert C, et al. Nivolumab in previously untreated melanoma without BRAF mutation. $N$ Engl J Med. 2015;372(4):320-330.

110. Lees CW, Ironside J, Wallace WA, Satsangi J. Resolution of non-small-cell lung cancer after withdrawal of anti-TNF therapy. $N$ Engl J Med. 2008;359(3):320-321.

111. Kong X, Sun T, Kong F, Du Y, Li Z. Chronic pancreatitis and pancreatic cancer. Gastrointest Tumors. 2014;1(3):123-134.

112. Cannarile MA, Weisser M, Jacob W, Jegg AM, Ries CH, Rüttinger D. Colony-stimulating factor 1 receptor (CSF1R) inhibitors in cancer therapy. JImmunother Cancer. 2017;5(1):53.

113. Farooqui M, et al. COX-2 inhibitor celecoxib prevents chronic morphine-induced promotion of angiogenesis, tumour growth, metastasis and mortality, without compromising analgesia. $\mathrm{Br} J$ Cancer. 2007;97(11):1523-1531.

114. Gordon JN, Trebble TM, Ellis RD, Duncan HD, Johns T, Goggin PM. Thalidomide in the treatment of cancer cachexia: a randomised placebo controlled trial. Gut. 2005;54(4):540-545.

115. Ruiz Garcia V, López-Briz E, Carbonell Sanchis R, Gonzalvez Perales JL, Bort-Marti S. Megestrol acetate for treatment of anorexiacachexia syndrome. Cochrane Database Syst Rev. 2013;(3):CD004310.

116. Ando K, et al. Tocilizumab, a proposed therapy for the cachexia of Interleukin6-expressing lung cancer. PLoS One. 2014;9(7):e102436.

117. Levitsky A, et al. Obesity is a strong predictor of worse clinical outcomes and treatment responses in early rheumatoid arthritis: results from the SWEFOT trial. RMD Open. 2017;3(2):e000458.

118. Castillo JJ, et al. Relationship between obesity and clinical outcome in adults with acute myeloid leukemia: a pooled analysis from four CALGB (alliance) clinical trials. Am J Hematol. 2016;91(2):199-204

119. Calle EE, Kaaks R. Overweight, obesity and cancer: epidemiological evidence and proposed mechanisms. Nat Rev Cancer. 2004;4(8):579-591.

120. Lyman GH. Impact of chemotherapy dose intensity on cancer patient outcomes. J Natl Compr Canc Netw. 2009;7(1):99-108.

121. Citron ML. Dose-dense chemotherapy: principles, clinical results and future perspectives. Breast Care (Basel). 2008;3(4):251-255.

122. Yakar $S$, et al. Increased tumor growth in mice with diet-induced obesity: impact of ovarian hormones. Endocrinology. 2006;147(12):5826-5834

123. Osman MA, Hennessy BT. Obesity correlation with metastases development and response to first-line metastatic chemotherapy in breast cancer. Clin Med Insights Oncol. 2015;9:105-112.

124.Engin A. Obesity-associated breast cancer: analysis of risk factors. Adv Exp Med Biol. 2017;960:571-606

125. Xue Y, et al. Hypoxia-independent angiogenesis in adipose tissues during cold acclimation. Cell Metab. 2009;9(1):99-109.

126. Bolinder J, Kerckhoffs DA, Moberg E, HagströmToft E, Arner P. Rates of skeletal muscle and adipose tissue glycerol release in nonobese and obese subjects. Diabetes. 2000;49(5):797-802.

127. Engin A. Adipose tissue hypoxia in obesity and its impact on preadipocytes and macrophages: hypoxia hypothesis. Adv Exp Med Biol. 2017;960:305-326

128. Mukhopadhyay D, Tsiokas L, Zhou XM, Foster D, Brugge JS, Sukhatme VP. Hypoxic induction of human vascular endothelial growth factor expression through c-Src activation. Nature. 1995;375(6532):577-581.

129. Cao Y. Tumor angiogenesis and molecular targets for therapy. Front Biosci (Landmark Ed). 2009;14:3962-3973.

130. Jain RK. Normalization of tumor vasculature: an emerging concept in antiangiogenic therapy. Science. 2005;307(5706):58-62.

131. Cao Y. Obesity protects cancer from drugs targeting blood vessels. Cell Metab. 2018;27(6):1163-1165.

132. Cao Y. Angiogenesis and vascular functions in modulation of obesity, adipose metabolism, and 
insulin sensitivity. Cell Metab. 2013;18(4):478-489.

133. Duong MN, et al. Adipose cells promote resistance of breast cancer cells to trastuzumabmediated antibody-dependent cellular cytotoxicity. Breast Cancer Res. 2015;17:57.

134. Behan JW, Avramis VI, Yun JP, Louie SG, Mittelman SD. Diet-induced obesity alters vincristine pharmacokinetics in blood and tissues of mice. Pharmacol Res. 2010;61(5):385-390.

135. Jain R, et al. Implications of obesity for drug therapy: limitations and challenges. Clin Pharmacol Ther. 2011;90(1):77-89.

136. Buechler C, Weiss TS. Does hepatic steatosis affect drug metabolizing enzymes in the liver? Curr Drug Metab. 2011;12(1):24-34.

137. Hanley MJ, Abernethy DR, Greenblatt DJ. Effect of obesity on the pharmacokinetics of drugs in humans. Clin Pharmacokinet. 2010;49(2):71-87.

138. Van der Veldt AA, et al. Rapid decrease in delivery of chemotherapy to tumors after anti-VEGF therapy: implications for scheduling of anti-angiogenic drugs. Cancer Cell. 2012;21(1):82-91.

139. Pan ST, Li ZL, He ZX, Qiu JX, Zhou SF. Molecular mechanisms for tumour resistance to chemotherapy. Clin Exp Pharmacol Physiol. 2016;43(8):723-737.

140. Doktorova H, Hrabeta J, Khalil MA, Eckschlager T. Hypoxia-induced chemoresistance in cancer cells: the role of not only HIF-1. Biomed Pap Med Fac Univ Palacky Olomouc Czech Repub. 2015;159(2):166-177.

141. Ganapathy-Kanniappan S, Geschwind JF. Tumor glycolysis as a target for cancer therapy: progress and prospects. Mol Cancer. 2013;12:152.

142.Harbuzariu A, Gonzalez-Perez RR. Leptin-Notch axis impairs 5-fluorouracil effects on pancreatic cancer. Oncotarget. 2018;9(26):18239-18253.

143. Cascetta P, et al. Pancreatic cancer and obesity: molecular mechanisms of cell transformation and chemoresistance. Int J Mol Sci. 2018;19(11):E3331.

144.Yu T, et al. Fusobacterium nucleatum promotes chemoresistance to colorectal cancer by modulating autophagy. Cell.2017;170(3):548-563.e16.

145. Yang Y, et al. Discontinuation of anti-VEGF cancer therapy promotes metastasis through a liver revascularization mechanism. Nat Commun. 2016;7:12680.

146.Okumura T, et al. Extra-pancreatic invasion induces lipolytic and fibrotic changes in the adipose microenvironment, with released fatty acids enhancing the invasiveness of pancreatic cancer cells. Oncotarget. 2017;8(11):18280-18295.

147. De Angel RE, et al. Stearoyl gemcitabine nanoparticles overcome obesity-induced cancer cell resistance to gemcitabine in a mouse postmenopausal breast cancer model. Cancer Biol Ther. 2013;14(4):357-364.

148. Chi M, et al. Adipocytes contribute to resistance of human melanoma cells to chemotherapy and targeted therapy. Curr Med Chem. 2014;21(10):1255-1267.

149. Bain GH, et al. Tumour expression of leptin is associated with chemotherapy resistance and therapy-independent prognosis in gastrooesophageal adenocarcinomas. Br JCancer. 2014;110(6):1525-1534.

150. Bartucci M, et al. Obesity hormone leptin induces growth and interferes with the cytotoxic effects of 5-fluorouracil in colorectal tumor stem cells. Endocr Relat Cancer. 2010;17(3):823-833.

151. Au Yeung CL, et al. Exosomal transfer of stroma-derived miR21 confers paclitaxel resistance in ovarian cancer cells through targeting APAF1. Nat Commun. 2016;7:11150.

152. Behan JW, et al. Adipocytes impair leukemia treatment in mice. Cancer Res. 2009;69(19):7867-7874.

153. Pramanik R, Sheng X, Ichihara B, Heisterkamp $\mathrm{N}$, Mittelman SD. Adipose tissue attracts and protects acute lymphoblastic leukemia cells from chemotherapy. Leuk Res. 2013;37(5):503-509.

154. Sheng X, et al. Adipocytes cause leukemia cell resistance to daunorubicin via oxidative stress response. Oncotarget. 2016;7(45):73147-73159.

155. Ehsanipour EA, et al. Adipocytes cause leukemia cell resistance to L-asparaginase via release of glutamine. Cancer Res. 2013;73(10):2998-3006.

156. Sheng X, Mittelman SD. The role of adipose tissue and obesity in causing treatment resistance of acute lymphoblastic leukemia. Front Pediatr. 2014;2:53.

157. Liu Z, et al. Mature adipocytes in bone marrow protect myeloma cells against chemotherapy through autophagy activation. Oncotarget. 2015;6(33):34329-34341.

158. Butturini AM, et al. Obesity and outcome in pediatric acute lymphoblastic leukemia. J Clin Oncol. 2007;25(15):2063-2069.

159. Amankwah EK, Saenz AM, Hale GA, Brown PA. Association between body mass index at diagnosis and pediatric leukemia mortality and relapse: a systematic review and meta-analysis. Leuk Lymphoma. 2016;57(5):1140-1148.

160. Orgel E, Genkinger JM, Aggarwal D, Sung L, Nieder M, Ladas EJ. Association of body mass index and survival in pediatric leukemia: a meta-analysis. Am J Clin Nutr. 2016;103(3):808-817.

161. Cao Y, Langer R. Optimizing the delivery of cancer drugs that block angiogenesis. Sci Transl Med. 2010;2(15):15ps3.

162. Motzer RJ, et al. Pazopanib versus sunitinib in metastatic renal-cell carcinoma. $N$ Engl JMed. 2013;369(8):722-731.

163. Meyerhardt JA, Mayer RJ. Systemic therapy for colorectal cancer. N EnglJMed. 2005;352(5):476-487.

164. Bergers G, Hanahan D. Modes of resistance to anti-angiogenic therapy. Nat Rev Cancer. 2008;8(8):592-603

165. Cao Y, Zhong W, Sun Y. Improvement of antiangiogenic cancer therapy by understanding the mechanisms of angiogenic factor interplay and drug resistance. Semin Cancer Biol. 2009;19(5):338-343.

166. Hedlund EM, et al. Tumor cell-derived placental growth factor sensitizes antiangiogenic and antitumor effects of anti-VEGF drugs. Proc Natl Acad Sci U S A. 2013;110(2):654-659.

167. Zhang D, et al. Antiangiogenic agents significantly improve survival in tumor-bearing mice by increasing tolerance to chemotherapy-induced toxicity. Proc Natl Acad Sci U S A. 2011;108(10):4117-4122.

168. Levine AJ, Puzio-Kuter AM. The control of the metabolic switch in cancers by oncogenes and tumor suppressor genes. Science. 2010;330(6009):1340-1344.
169. Choi J, Cha YJ, Koo JS. Adipocyte biology in breast cancer: from silent bystander to active facilitator. Prog Lipid Res. 2018;69:11-20.

170.Park JH, et al. Fatty acid oxidation-driven src links mitochondrial energy reprogramming and oncogenic properties in triple-negative breast cancer. Cell Rep. 2016;14(9):2154-2165.

171. Guiu B, et al. Visceral fat area is an independent predictive biomarker of outcome after first-line bevacizumab-based treatment in metastatic colorectal cancer. Gut. 2010;59(3):341-347.

172. Ladoire S, et al. Visceral fat area as a new independent predictive factor of survival in patients with metastatic renal cell carcinoma treated with antiangiogenic agents. Oncologist. 2011;16(1):71-81.

173. Trédan O, et al. Angiogenesis and tumor microenvironment: bevacizumab in the breast cancer model. Target Oncol. 2015;10(2):189-198.

174. Steffens S, et al. Does obesity influence the prognosis of metastatic renal cell carcinoma in patients treated with vascular endothelial growth factor-targeted therapy? Oncologist. 2011;16(11):1565-1571.

175. Crawford Y, et al. PDGF-C mediates the angiogenic and tumorigenic properties of fibroblasts associated with tumors refractory to anti-VEGF treatment. Cancer Cell. 2009;15(1):21-34.

176. Casanovas O, Hicklin DJ, Bergers G, Hanahan D. Drug resistance by evasion of antiangiogenic targeting of VEGF signaling in late-stage pancreatic islet tumors. Cancer Cell. 2005;8(4):299-309.

177. Maniati E, Hagemann T. IL-17 mediates resistance to anti-VEGF therapy. Nat Med. 2013;19(9):1092-1094.

178. Datta K, Suman S, Kallakury BV, Fornace AJ Exposure to heavy ion radiation induces persistent oxidative stress in mouse intestine. PLoS One. 2012;7(8):e42224.

179. Yamamori $T$, et al. Ionizing radiation induces mitochondrial reactive oxygen species production accompanied by upregulation of mitochondrial electron transport chain function and mitochondrial content under control of the cell cycle checkpoint. Free Radic Biol Med. 2012;53(2):260-270.

180. Kamat JP, Devasagayam TP. Oxidative damage to mitochondria in normal and cancer tissues, and its modulation. Toxicology. 2000;155(1-3):73-82.

181. Iyer A, Fairlie DP, Prins JB, Hammock BD, Brown L. Inflammatory lipid mediators in adipocyte function and obesity. Nat Rev Endocrinol. 2010;6(2):71-82.

182. Manna P, Jain SK. Obesity, oxidative stress, adipose tissue dysfunction, and the associated health risks: causes and therapeutic strategies. Metab Syndr Relat Disord. 2015;13(10):423-444

183. Furukawa S, et al. Increased oxidative stress in obesity and its impact on metabolic syndrome. JClin Invest. 2004;114(12):1752-1761.

184.Engin A. The pathogenesis of obesity-associated adipose tissue inflammation. Adv Exp Med Biol. 2017;960:221-245

185. Le Sage F, Meilhac O, Gonthier MP. Porphyromonas gingivalis lipopolysaccharide induces pro-inflammatory adipokine secretion and oxidative stress by regulating Toll-like receptor-mediated signaling pathways and redox enzymes in adipocytes. Mol Cell Endocrinol. 2017;446:102-110.

186.Gonzalez FJ, Xie C, Jiang C. The role of hypoxia- 
inducible factors in metabolic diseases. Nat Rev Endocrinol. 2018;15(1):21-32.

187. Norouzirad R, González-Muniesa P, Ghasemi A. Hypoxia in obesity and diabetes: potential therapeutic effects of hyperoxia and nitrate. Oxid Med Cell Longev. 2017;2017:5350267.

188. Divella R, De Luca R, Abbate I, Naglieri E, Daniele A. Obesity and cancer: the role of adipose tissue and adipo-cytokines-induced chronic inflammation. J Cancer. 2016;7(15):2346-2359.

189. Ryan S. Adipose tissue inflammation by intermittent hypoxia: mechanistic link between obstructive sleep apnoea and metabolic dysfunction. JPhysiol (Lond). 2017;595(8):2423-2430.

190.Coelho P, et al. Adipocyte secretome increases radioresistance of malignant melanocytes by improving cell survival and decreasing oxidative status. Radiat Res. 2017;187(5):581-588.
191. Singh S, et al. Mesenchymal stem cells show radioresistance in vivo. J Cell Mol Med. 2012;16(4):877-887.

192.Short MW, Burgers KG, Fry VT. Esophageal cancer. Am Fam Physician. 2017;95(1):22-28.

193. Cook MB, et al. Pathogenesis and progression of oesophageal adenocarcinoma varies by prior diagnosis of Barrett's oesophagus. Br JCancer. 2016;115(11):1383-1390.

194. Shridhar R, et al. Radiation therapy and esophageal cancer. Cancer Control. 2013;20(2):97-110.

195. Yang HY, et al. IGF-1 from adipose-derived mesenchymal stem cells promotes radioresistance of breast cancer cells. Asian Pac J Cancer Prev. 2014;15(23):10115-10119.

196. Gennari A, et al. Impact of body mass index (BMI) on the prognosis of high-risk early breast cancer (EBC) patients treated with adjuvant chemother- apy. Breast Cancer Res Treat. 2016;159(1):79-86.

197. Karatas F, et al. Obesity is an independent prognostic factor of decreased pathological complete response to neoadjuvant chemotherapy in breast cancer patients. Breast. 2017;32:237-244.

198. Arce-Salinas C, et al. Overweight and obesity as poor prognostic factors in locally advanced breast cancer patients. Breast Cancer Res Treat. 2014;146(1):183-188.

199. Bougaret L, et al. Adipocyte/breast cancer cell crosstalk in obesity interferes with the antiproliferative efficacy of tamoxifen. PLoS One. 2018;13(2):e0191571.

200.Griner SE, Wang KJ, Joshi JP, Nahta R. Mechanisms of adipocytokine-mediated trastuzumab resistance in HER2-positive breast cancer cell lines. Curr Pharmacogenomics Person Med. 2013;11(1):31-41. 